\title{
Observations of Galactic Magnetic Fields
}

\author{
Rainer Beck \\ Max-Planck-Institut für Radioastronomie, D-53121 Bonn, Germany
}

\begin{abstract}
Magnetic fields are anchored in gas clouds. Field lines are tangled in spiral arms, but highly regular between the arms. The similarity of pitch angles between gaseous and magnetic arms suggests a coupling between the density wave and the magnetic wave. Observations of large-scale patterns in Faraday rotation favour a dynamo origin of the regular fields. Fields in barred galaxies do not reveal the strong shearing shocks observed in the cold gas, but swing smoothly from the upstream region into the bar. Magnetic fields are important for the dynamics of gas clouds, for the formation of spiral structures, bars and halos, and for mass and angular momentum transport in central regions.
\end{abstract}

\section{Introduction}

Linearly polarized radio continuum emission and its Faraday rotation provide information on interstellar magnetic fields in external galaxies (Beck et al. 1996; Beck 2000) and in the Galactic center. Polarization surveys of the Galactic plane show the fine structure of the local Galactic field (Wielebinski, this Proceedings) while Faraday rotation measures of pulsars and extragalactic sources give information about the large-scale structure (Reid, this Proceedings).

\section{Equipartition Field Strengths: Reliable?}

The average strength of the total $\left\langle B_{t, \perp}\right\rangle$ and the resolved regular field $\left\langle B_{r e g, \perp}\right\rangle$ in the plane of the sky can be derived from the total and polarized radio synchrotron intensity, respectively, if energy-density equipartition between cosmic rays and magnetic fields is assumed. Average equipartition strengths of the total field range between $\left\langle B_{t}\right\rangle \simeq 6 \mu \mathrm{G}$ in radio-faint spiral galaxies like M31 and M33, and $\simeq 15 \mu \mathrm{G}$ in grand-design galaxies like M51, M83 and NGC 6946. In spiral arms the total field strengths can reach $\simeq 20 \mu \mathrm{G}$, and even more in central regions.

In the Galaxy the accuracy of equipartition can be tested because we have independent information about the local cosmic-ray electrons. Analysis of the radio synchrotron emission, the local cosmic-ray electron density and diffuse continuum $\gamma$-rays yields a local strength of the total field of $6 \pm 1 \mu \mathrm{G}$ (Strong et al. 2000 ), almost the same value as derived from energy equipartition (Berkhuijsen, in Beck et al. 1996). Even the radial variation of the equipartition field strength in the Galaxy is in excellent agreement with that derived by Strong et al. (2000). 
Rotation and emission measures from pulsars yield an average strength of the local regular (interarm) field of $\simeq 1.8 \mu \mathrm{G}$, lower than derived from synchrotron and optical polarization data (Heiles 1996) and lower than values in external galaxies. If the fluctuations in magnetic field and in thermal electron density are anticorrelated, pulsar data underestimate the regular field strength.

\section{Total Fields: Anchored in Gas Clouds}

Total synchrotron intensities are highest in spiral arms, but the degree of polarization is low, probably due to tangling of the field lines in star-forming regions. The field in spiral arms is mostly irregular.

Comparison of the maps of the total radio emission and the far-infrared dust emission (ISOPHOT) reveals a surprisingly close connection. In the galaxy M83 the correlation is valid even in the mid-infrared (ISOCAM) on a spatial scale of $500 \mathrm{pc}$ (Vogler et al., in prep.). This result cannot be explained by the distribution of cosmic rays produced in star-forming regions. The time delay between massive stars forming from gas clouds and the acceleration of cosmic rays in supernova remnants as well as diffusion of cosmic rays would smear out the radio image. Instead, total radio intensity depends mainly on the strength of the total magnetic fields. Magnetic fields are obviously anchored in (warm?) gas clouds which are traced by the warm dust. This can explain the correlation between radio and infrared intensities (Niklas \& Beck 1997; Hoernes et al. 1998). The total field is strong in spiral arms because the number density of gas clouds is high there so that more magnetic flux can be anchored.

Note that the turbulent dynamo (Subramanian, this Proceedings) may also be able to generate small-scale fields with strengths limited by gas density.

\section{Regular Interarm Fields: Dynamo or Slow MHD Waves?}

The strongest regular fields (traced by polarized radio intensity) occur in interarm regions of spiral galaxies, in some cases concentrated in "magnetic arms" (e.g. in M83, IC 342, NGC 2997 and NGC 6946). This cannot be explained by less field tangling or less Faraday depolarization in these regions (Beck \& Hoernes 1996). The fields in the "magnetic arms" are almost totally regular. The peak equipartition strength of the interarm field in NGC 6946 is $\simeq 13 \mu G$.

The similarity of pitch angle of the spiral arms and the magnetic (inter)arms suggests a coupling between the density and the magnetic wave. Fan \& Lou (1997) proposed slow MHD waves which propagate in an almost rigidly rotating disk, with the maxima in field strength phase-shifted against those in gas density. Han et al. (1999) found a correlation between the magnetic arms and interarm gas features generated in numerical models of perturbed galactic disks (which, however, neglect the effect of magnetic fields). In dynamo models, using the assumption that the local dynamo number is larger between the optical arms than in the arms, magnetic arms evolve between the optical arms in a differentially rotating disk (Elstner, this Proceedings). However, the back-reaction of the field onto the gas has not yet been considered in dynamo models. 


\section{Faraday Rotation: Footprints of Dynamo Action}

The $B$-vectors of linearly polarized emission just indicate anisotropy of the magnetic field distribution, e.g. compressed (incoherent) fields. Maps of Faraday rotation measures (RMs) are necessary to reveal coherent fields, as observed in M31 (Beck 1982, 2000), NGC 6946 and NGC 2997 (Han et al. 1999).

Dynamos are promising candidates to generate coherent fields. The linear mean-field dynamo (Elstner, this Proceedings; Moss, this Proceedings) generates magnetic field modes which can be distinguished by large-scale patterns in RM maps. Radio observations of M31 revealed a $20 \mathrm{kpc}$-sized torus of coherent axisymmetric fields (Beck 1982, 2000). In M31 and a few other galaxies with a dominating axisymmetric mode the field points inwards everywhere which cannot be explained by dynamo action (Krause \& Beck 1998).

In most spiral galaxies a superposition of dynamo modes is necessary to describe the observations (Beck 2000). Field reversals like those detected in our Galaxy (see Reid, this Proceedings) can be generated by such a superposition. Surprisingly, nothing similar was found in RM maps of external galaxies.

\section{Fields in Barred Galaxies: Going with the Flow?}

Gas and stars in barred galaxies move in highly noncircular orbits. Streamlines are strongly deflected in the bar region along shock fronts, behind which the cold gas is compressed in a fast shearing flow. In a survey of 20 barred galaxies (Shoutenkov et al., in preparation) ridges of enhanced total and regular magnetic fields generally coincide well with the optical dust lanes. A general similarity of the $B$-vectors e.g. in NGC 1097 (Beck et al. 1999) and gas streamlines around the bar obtained in simulations suggests that the field is aligned with the flow.

In several barred galaxies strong polarized emission emerges also from the upstream regions, separated from the bar by a strip of zero polarized intensity where the observed $B$-vectors change their orientation abruptly, leading to depolarization. The deflection regions of the field are located 1-3 kpc in front of the dust lanes, in contrast to conditions in classical shocks. Recent high-resolution VLA observations of NGC 1097 revealed a fast, but smooth change of the field's orientation. Apparently the field is not shocked, e.g. because it is coupled to the warm ISM phase. However, the field orientations may also deviate from the upstream gas flow. No models of magnetized shearing shocks exist yet.

\section{Central Fields: Feeding the Nucleus?}

The circumnuclear ring of NGC $1097(\simeq 1.5 \mathrm{kpc}$ diameter) is a site of ongoing intense star formation, with an active nucleus in its centre. The local equipartition strengths of the total and regular magnetic fields are $B_{t} \simeq 40 \mu \mathrm{G}$ and $B_{r} \simeq 7 \mu \mathrm{G}$ in the ring (Beck et al. 1999). Conditions for dynamo action are ideal in the ring. The structure of the innermost field is spiral and agrees with that of optical dust filaments. Magnetic stress in the circumnuclear ring can drive mass inflow, sufficient to feed the active nucleus in NGC 1097.

Spiral fields are observed in the central regions of all nearby galaxies and may play an important role for mass and angular momentum transport. 


\section{Halo Fields: Supporting the Outflow?}

Most edge-on galaxies possess thick radio disks with $\simeq 1-2 \mathrm{kpc}$ scale heights and field orientations mainly parallel to the disk (Dumke et al. 1995). Bright, extended radio halos with dominating vertical field components are rare (NGC 4631, NGC 4666 and M82; central region of the Galaxy). Magnetic spurs in these halos are connected to star-forming regions in the disk, probably dragged out by a strong galactic wind (Golla \& Hummel 1994). On the other hand, a strong dynamo-generated field may support the formation of a wind. The field in the halo of NGC 4631 has a dipolar structure, signature of an antisymmetric dynamo mode, but this has to be confirmed by future RM data.

\section{The Future: What Do We Need?}

Systems operating at $\lambda 13 \mathrm{~cm} / 2.3 \mathrm{GHz}$ will improve polarization studies of Faraday-thin galaxies and their halos. Such systems are now available at the WSRT (Westerbork/NL) and hopefully soon at the VLA. For Faraday-thick galaxies and central regions systems at very high frequencies are required. A multihorn system at $\lambda 1 \mathrm{~cm} / 30 \mathrm{GHz}$ is planned for the Effelsberg $100-\mathrm{m}$ telescope.

The collecting areas of the present-day radio telescopes are too small. A $5 \mu \mathrm{G}$ regular magnetic field gives a polarized intensity at $\lambda 6 \mathrm{~cm}$ of only about $0.2 \mu \mathrm{Jy}$ per 1" beam. The planned Square Kilometer Array will provide sufficient sensitivity to unravel the fine structure of interstellar fields and to observe pulsar rotation measures and Zeeman splitting in external galaxies.

\section{References}

Beck, R. 1982, A\&A, 106, 121

Beck, R. 2000, Phil. Trans. R. Soc. Lond. A, 358, 777

Beck, R., \& Hoernes, P. 1996, Nature, 379, 47

Beck, R., Brandenburg, A., Moss, D., Shukurov, A., \& Sokoloff, D. 1996, ARA\&A, 34, 155

Beck, R., Ehle, M., Shoutenkov, V., Shukurov, A., \& Sokoloff, D. 1999, Nature, 397,324

Dumke, M., Krause, M., Wielebinski, R., \& Klein, U. 1995, A\&A, 302, 691

Fan, Z., \& Lou, Y.Q. 1997, MNRAS, 291, 91

Golla, G., \& Hummel, E. 1994, A\&A, 284, 777

Han, J.L., Beck, R., Ehle, M., Haynes, R.F., \& Wielebinski, R. 1999, A\&A, 348, 405

Heiles, C. 1996, in ASP Conf. Ser. Vol. 97, Polarimetry of the Interstellar Medium, eds. W. G. Roberge \& D. C. B. Whittet (eds.), 457

Hoernes, P., Berkhuijsen, E.M., \& Xu, C. 1998, A\&A, 334, 57

Krause, F., \& Beck, R. 1998, A\&A, 335, 789

Niklas, S., \& Beck, R. 1997, A\&A, 320, 54

Strong, A.W., Moskalenko, I.V., \& Reimer, O. 2000, ApJ, 537, 763 